\title{
Prophylactic mechanical hemodynamic support for catheter ablation of VT: locking the barn door before the horse has bolted
}

\author{
Pasquale Santangeli ${ }^{1}$ (D) $\cdot$ Luigi Di Biase ${ }^{2}$
}

Received: 18 September 2016 / Accepted: 16 November 2016/Published online: 25 November 2016

(C) Springer Science+Business Media New York 2016

In patients with recurrent drug-refractory ventricular tachycardia (VT) in the setting of structural heart disease, radiofrequency catheter ablation (RFCA) has emerged as an important therapeutic strategy to achieve VT suppression and long-term arrhythmia control [1]. In recent years, significant improvements in the techniques and technologies available for RFCA have been paralleled by an increasing number of procedures performed in high-risk and complex patient subsets [1-3]. In these subjects, the competing risks associated with the concomitant presence of advanced heart failure syndromes and high burden of associated comorbidities pose substantial periprocedural and post-procedural management challenges $[2,3]$. In particular, the occurrence of periprocedural acute hemodynamic decompensation (AHD) is a major concern due to its significant association with post-procedural mortality [4]. Each aspect of a VT ablation procedure including induction of anesthesia, programmed ventricular stimulation, presence of spontaneous, and/or mechanically induced VT attempts at pace termination or needs for electrical cardioversions, and fluid overload during mapping and ablation incurs in a potential risk of precipitating AHD resulting in severe end-organ (e.g. brain, kidney, liver) hypoperfusion, and effective therapeutic strategies to prevent the occurrence of this complication are highly warranted.

Pasquale Santangeli

pasquale.santangeli@uphs.upenn.edu

1 Electrophysiology Section, Cardiovascular Division, Hospital of the University of Pennsylvania, 9 Founders Pavilion - Cardiology, 3400 Spruce St, Philadelphia, PA 19104, USA

2 Montefiore Hospital, Albert Einstein College of Medicine, New York, NY, USA
In this issue of JICE, Mathuria et al. report the results of an elegant and well-designed observational study assessing the outcomes of pre-emptive versus rescue use of percutaneous left ventricular assist device (P-LVAD) in a group of 93 patients with structural heart disease undergoing VT ablation [5]. Overall, 36/ 93 (39\%) patients received a P-LVAD, with 12 undergoing rescue P-LVAD placement due to periprocedural AHD and the remaining 24 patients who received a P-LVAD prophylactically before the ablation procedure. The patients receiving a P-LVAD were compared with a control group of 57 patients undergoing VT ablation during the same study period and without insertion of a P-LVAD. Interestingly, the authors reported a strikingly higher 30-day mortality rate in the patients who underwent rescue P-LVAD (58\%) compared to that in both the pre-emptive PLVAD (4\%) and no P-LVAD (3\%) group. Notably, the patients who underwent rescue P-LVAD had a similar risk profile compared to patients who had prophylactic placement of the mechanical support devices. In this regard, the authors used the PAINESD risk score to evaluate differences in clinical profile and risk of periprocedural AHD in their patients (Fig. 1). The PAINESD risk score has been developed by the University of Pennsylvania group in an observational study including 193 consecutive patients undergoing catheter ablation of VT in the setting of structural heart disease [4]. Similar to the findings by Mathuria et al., periprocedural AHD requiring emergent placement of mechanical support devices and/or premature procedure discontinuation was found to have a strong negative prognostic impact, with an up to $50 \%$ mortality after an average follow-up of $21 \pm$ 7 months. The predictors of AHD that were incorporated in the PAINESD risk score included age, diabetes, ischemic cardiomyopathy, reduced left ventricular ejection fraction, chronic obstructive pulmonary disease (COPD), presentation with VT storm, and NYHA functional class III/IV. An additional variable that was also found to be associated with increased risk of AHD was the use of general anesthesia [4]. 


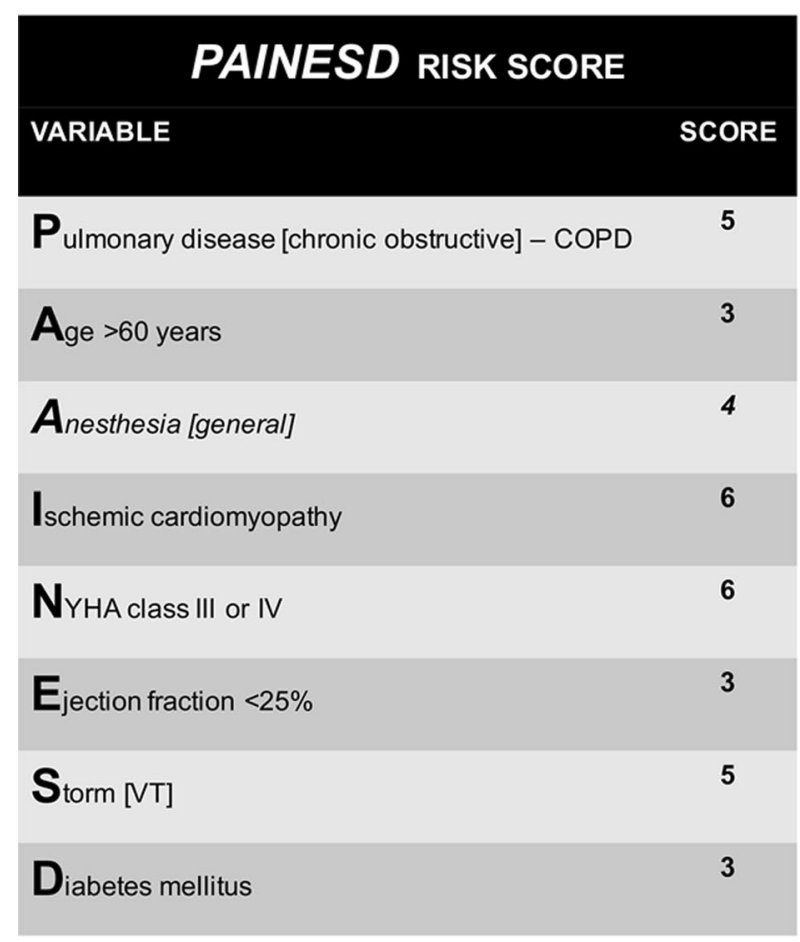

Fig. 1 PAINESD risk score to predict periprocedural acute hemodynamic decompensation in patients undergoing VT ablation. The risk score has been developed with and without the variable "general anesthesia" because its use may be discretional and variable across

Importantly, the PAINESD score appeared to perform well in the independent patient cohort reported by Mathuria et al. [5]. The patients who received a P-LVAD had a significantly higher score compared to the control group that did not undergo PLVAD implantation, and there was no difference in score between the patients who underwent rescue versus pre-emptive PLVAD insertion (mean score 17.8 in the rescue P-LVAD group, 16.5 in the prophylactic P-LVAD group, and 13.4 in the control group). Overall, this study adds an important piece of evidence to prior studies and supports a role for prophylactic P-LVAD insertion in patients with high PAINESD scores in order to improve post-procedural mortality. The key novel information is that PLVAD may provide incremental benefit only when used preemptively (i.e., before the clinical occurrence of AHD), with limited role if used as rescue intervention. In other words, whenever periprocedural AHD has occurred, it may be too late to revert the vicious pathologic process that ultimately leads to post-procedural mortality.

From a mechanistic standpoint, prophylactic use of P-LVAD offers many advantages that may help preventing AHD, including maintenance of vital organ perfusion; reduction of intracardiac filling pressures; reduction of LV volumes, wall stress, and myocardial consumption of oxygen; and support of systemic circulation and reduction of cardiac stunning due to multiple VT inductions for mapping and during ablation [6, 7]. The ventricular unloading effect with P-LVAD may play a crucial role
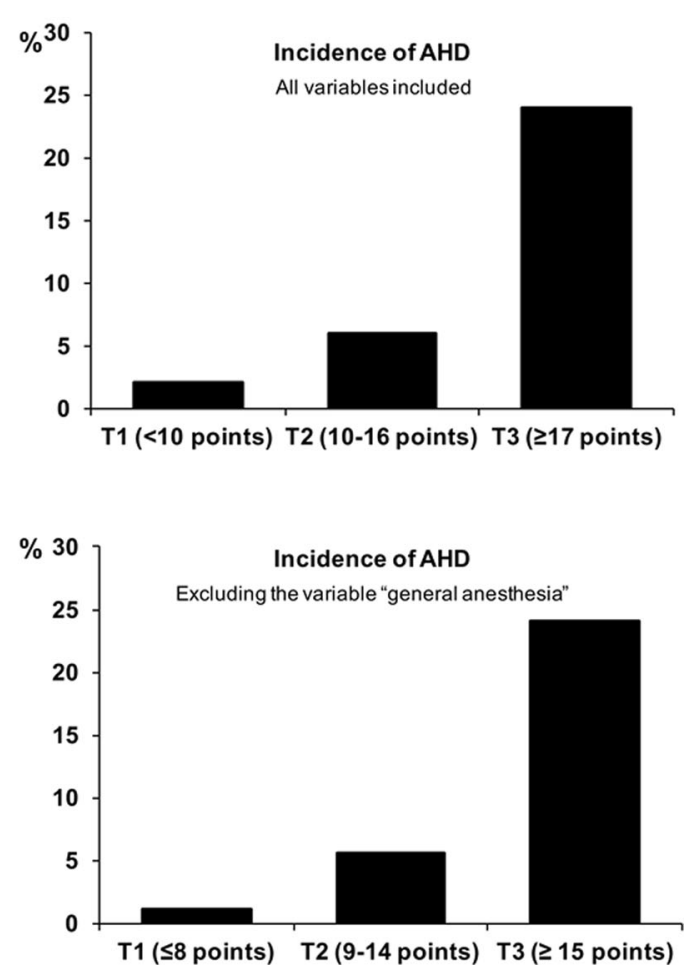

different institutions. Increasing tertiles of risk score are associated with progressively higher risk of acute hemodynamic decompensation. Modified from Santangeli et al. [4]

also in patients with large substrates undergoing a purely substrate-based ablation approach. In these patients, prolonged procedural (and anesthesia) times due to the necessity for complete substrate ablation and the resulting periprocedural fluid overload may precipitate AHD [4].

An important aspect that was not completely investigated by Mathuria et al. concerns the choice of the optimal mechanical support device in the individual patient deemed at high risk of periprocedural AHD. The authors used only the Impella ${ }^{\mathrm{TM}}$ (Abiomed, Inc., Danvers, MA, USA) and the TandemHeart ${ }^{\mathrm{TM}}$ (CardiacAssist, Inc., Pittsburgh, PA, USA) devices, which provide only LV support. In patients with advanced heart failure and VT, severe LV dysfunction is frequently accompanied by significant RV dysfunction which predicts poor response to LV assist devices like Impella ${ }^{\mathrm{TM}}$ or TandemHeart ${ }^{\mathrm{TM}}$. In these cases, our experience suggests better results with veno-arterial extracorporeal membrane oxygenation (VA-ECMO). Direct clinical comparative studies between different mechanical support devices are lacking, although some pre-clinical data exist. Ostadal et al. compared the hemodynamic responses to sustained $\mathrm{VF}$ or rapid ventricular pacing (200 and $300 \mathrm{bpm}$ ) with VA-ECMO, the Impella $^{\mathrm{TM}}$ and TandemHeart ${ }^{\mathrm{TM}}$ in a pig model [8]. The study endpoint was maintenance of a target mean arterial pressure (MAP) of 70-80 mmHg. With ventricular pacing at $200 \mathrm{bpm}$, no difference in mean arterial pressure was observed between the three MHS systems; however, with ventricular pacing at 
300 bpm and during sustained VF, only ECMO support was able to maintain the target MAP, with the lowest efficacy being observed with the Impella ${ }^{\mathrm{TM}}$ device [8]. Therefore, whether the results reported by Mathuria et al. can be generalized also to patients undergoing VA-ECMO support warrant further investigation.

In summary, we believe that the report by Mathuria et al. should be taken into serious account by the electrophysiology community involved in the care of patients with advanced heart failure and recurrent VT. Notwithstanding the observational study design, the results confirm and expand prior findings from independent patient cohorts and are striking. Periprocedural AHD is convincingly emerging as a worrisome complication of catheter ablation of VT in patients with structural heart disease, and prevention of this complication may result in improved mortality. A simple risk score (PAINESD) has now been reproducibly shown able to identify patients at risk of AHD. In these patients, prophylactic mechanical hemodynamic support may lock the barn door before the horse has bolted, and improve post-procedural outcomes.

\section{Compliance with ethical standards}

Funding sources None.

Disclosures None related to this manuscript.

\section{References}

1. Aliot EM, Stevenson WG, Almendral-Garrote JM, Bogun F, Calkins $\mathrm{CH}$, Delacretaz E, et al. EHRA/HRS Expert
Consensus on Catheter Ablation of Ventricular Arrhythmias: developed in a partnership with the European Heart Rhythm Association (EHRA), a Registered Branch of the European Society of Cardiology (ESC), and the Heart Rhythm Society (HRS); in collaboration with the American College of Cardiology (ACC) and the American Heart Association (AHA). Heart Rhythm. 2009;6:886-933.

2. Stevenson WG, Wilber DJ, Natale A, Jackman WM, Marchlinski FE, Talbert T, et al. Irrigated radiofrequency catheter ablation guided by electroanatomic mapping for recurrent ventricular tachycardia after myocardial infarction: the multicenter thermocool ventricular tachycardia ablation trial. Circulation. 2008;118:2773-82.

3. Tung R, Josephson ME, Reddy V, Reynolds MR, Investigators S-V. Influence of clinical and procedural predictors on ventricular tachycardia ablation outcomes: an analysis from the substrate mapping and ablation in Sinus Rhythm to Halt Ventricular Tachycardia Trial (SMASH-VT). J Cardiovasc Electrophysiol. 2010;21:799-803.

4. Santangeli P, Muser D, Zado ES, Magnani S, Khetpal S, Hutchinson $\mathrm{MD}$, et al. Acute hemodynamic decompensation during catheter ablation of scar-related ventricular tachycardia: incidence, predictors, and impact on mortality. Circ Arrhythm Electrophysiol. 2015;8:68-75.

5. Mathuria N, Wu G, Rojas-Delgado F, Shuraih M, Razavi M, Civitello A, et al. Outcomes of pre-emptive and rescue use of percutaneous left ventricular assist device in patients with structural heart disease undergoing catheter ablation of ventricular tachycardia, J. Interv. Card. Electrophysiol. 2016. doi:10.1007/s10840-016-0168-8.

6. Reddy YM, Chinitz L, Mansour M, Bunch TJ, Mahapatra S, Swarup $\mathrm{V}$, et al. Percutaneous left ventricular assist devices in ventricular tachycardia ablation: multicenter experience. Circ Arrhythm Electrophysiol. 2014;7:244-50.

7. Miller MA, Dukkipati SR, Mittnacht AJ, Chinitz JS, Belliveau L, Koruth JS, et al. Activation and entrainment mapping of hemodynamically unstable ventricular tachycardia using a percutaneous left ventricular assist device. J Am Coll Cardiol. 2011;58:1363-71.

8. Ostadal P, Mlcek M, Holy F, Horakova S, Kralovec S, Skoda J, et al. Direct comparison of percutaneous circulatory support systems in specific hemodynamic conditions in a porcine model. Circ Arrhythm Electrophysiol. 2012;5:1202-6. 